\title{
Adsorption Processes Coupled with Mass Transport at Macro-electrodes: New Insights from Simulation
}

\author{
Minjun Yang1, Richard G. Compton ${ }^{1}\left(\Xi_{\Theta}\right)$ \\ ${ }^{1}$ Department of Chemistry, Physical and Theoretical Chemistry Laboratory, University of Oxford, South Parks \\ Road, Oxford OX1 3QZ, United Kingdom
}

\begin{abstract}
A general simulation model is developed for voltammetry of species adsorbed from solution involving coupled mass transport and adsorption which distinguishes the relative contributions from the adsorption kinetics, the mass transport and electron transfer kinetics of the reactants and products. The model assumes only the adsorbed species are electrochemically active. To provide meaningful insights, we focus on three limiting cases in which the fate of the formed product differs. In Case One, the product remains on the surface and does not desorb; in Case Two, the product desorbs slowly into solution; in Case Three, the product desorbs rapidly into solution. In each case, the effect of independent parameters such as the adsorption rate constants, the electron transfer kinetics and the voltage scan rate are investigated. New insights from this simulation includes a steady state voltage-current response for a macro-electrode when rate determining step becomes 'bottlenecked' by the slow desorption of the product. Furthermore, when the rate of adsorption and desorption of the reaction and product are fast compared to the mass transport and are not rate determining, the simulated current for the redox process via the adsorption pathway can be ca. 2.3 times higher to that when electron transfer occurs via the solution phase.
\end{abstract}

Keywords

Adsorption, simulation, electrochemistry, macroelectrodes, cyclic voltammetry 


\section{Introduction}

In analytical electrochemistry, it is common for redox active species to adsorb onto the electrode surface ${ }^{1-5}$ The naturally occurring redox active organic compounds responsible for the characteristic taste of garlic, ${ }^{6}$ ginger $^{7}$ and chilli $^{8}$ have been electrochemically targeted and analysed for the 'strength' or 'hotness' of its corresponding organic matter. The redox active organic compounds targeted were thiosulfinates, gingerol and capsaicin respectively; in all cases, they were all found to adsorb onto the surface of the electrode. Thus understanding the roles of adsorption in voltammetry is vital for analytical electrochemistry as the voltammetric shape and current response can be affected.

The pioneering and seminal work by Laviron and co-workers have contributed extensively to the understanding of electrochemical processes that occur on an electrode surface, especially when both the reactants and products are strongly adsorbed - which is often the case on mercury electrodes. ${ }^{9-11}$ The work includes, but is not limited to: simulation of various type of EC reactions (electrochemical reactions follow by chemical reactions) on the electrode surface, ${ }^{12-17}$ mathematical theory for the understanding of role of adsorption on rotating disk voltammetry assuming a fast and non-rate-determining adsorption step; ${ }^{18,19}$ alternating current polarography and a Faradaic impedance study of strongly adsorbed electroactive species..$^{20-23}$

More recent development in this field includes the modelling of one-step electrodeposition reaction by Pekka et al. The work investigates the linear sweep voltammetry of the soluble-insoluble system with the generation of working curves which were used to extract kinetic information of the $\mathrm{Cu}_{(\mathrm{aq})}^{+} / \mathrm{Cu}_{(\mathrm{s})}$ redox couple in acetonitrile. ${ }^{24}$ Furthermore, the simulation by Chevallier et al. considers the adsorption kinetics of the reactant coupled with mass transport, where the product remains adsorbed on the surface. ${ }^{25}$ This model examined all six of the independent control parameters and investigated how these affect the voltammetric shape; the six independent parameters studied were voltage scan rate, adsorption rate constant, equilibrium constant, standard electron transfer rate constant, saturation parameter and the initial coverage of species. The theory explained the adsorption behaviour observed during the reduction of aqueous $\left[\mathrm{Co}\left(\mathrm{NH}_{3}\right)_{6}\right]^{3+}$ at fast voltage scan rates. ${ }^{26}$

This work presents a general model for voltammetry which includes adsorbed species. The work advances from Chevallier et al. by considering the kinetics of adsorption and desorption of both the reactant and product, the electron transfer kinetics and the mass transport to and from the bulk solution. Herein, we assume only the adsorbed species are electrochemically active. The model 
contains a total of nine independent parameters from which is obviously impossible to produce full working surfaces considering all of the parameters! To provide a meaningful insight of how the adsorption kinetics affect the current-voltage curve, the simulation focuses on three limiting cases in which the 'fate' of the product differs. In Case One, the product remains on the surface and does not desorb; in Case Two, the product slowly desorbs into solution and in Case Three, the product desorbs rapidly into solution. For each cases, the effect of independent parameters such as the adsorption rate constant of the reactant, the electron transfer kinetics and the voltage scan rate are investigated.

\section{Theory}

This section introduces the theoretical model employed for the simulations. The simulation considers only the surface bound species to be electrochemically active. That is the solution species must adsorb prior to electron transfer.

\subsection{Theoretical model}

The simulation model is summarized in Figure 1. The model contains two solution phase species $A$ and $B$ in the bulk solution with concentrations $[A]_{b u l k}$ and $[B]_{b u l k}$. The surface concentrations of the species at the electrode-solution interface are $[A]_{0}$ and $[B]_{0}$, from where they can adsorb onto the electrode with an adsorption rate constant of $k_{a d s}^{A / B}\left(\mathrm{~m}^{3} \mathrm{~mol}^{-1} \mathrm{~s}^{-1}\right)$. The surface bound species can either undergo redox reaction with a standard electrochemical rate constant $k_{0}$ or desorb into solution with a desorption rate constant of $k_{d e s}^{A / B}\left(\mathrm{~s}^{-1}\right)$.

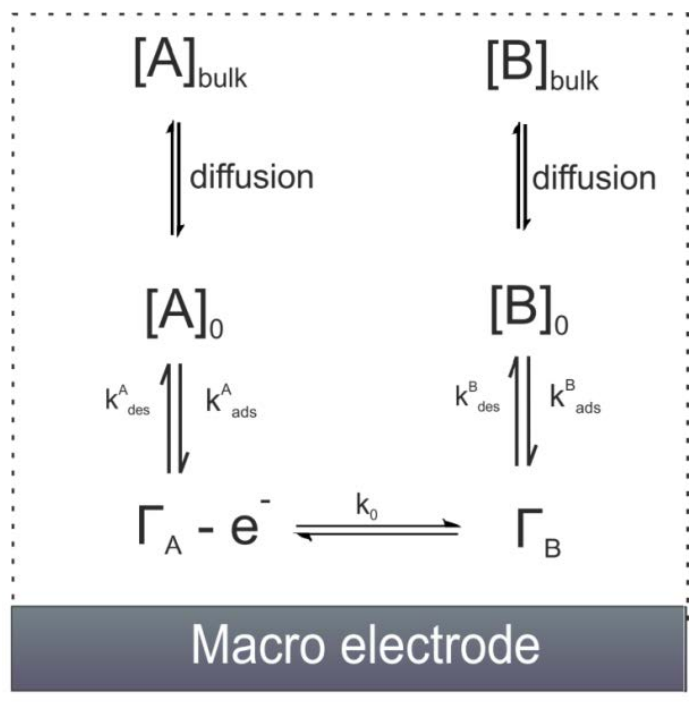

Figure 1. Schematic of the simulation model. 
The simulation model adopts the Langmuir adsorption isotherm, ${ }^{27}$ which is a limiting case of the Frumkin's adsorption isotherm ${ }^{28,29}$ when the interfacial particle-particle interaction parameter $\alpha$ is equal to zero, i.e. the surface-bound species are assumed non-interacting. Furthermore, the number of adsorption sites and the binding are assumed independent of the applied potential and the unoccupied sites are presumed competitively accessible to both adsorbates until a maximum surface coverage $\Gamma_{\max }\left(\mathrm{mol} \mathrm{m}^{-2}\right)$ is reached, with one adsorbate occupies one site. The simulation assumes only the adsorbed $A$ and $B$ are electrochemically active as indicated in Figure 1. The time-dependant equations that describe the rate of adsorption and mass transport of $A$ and $B$ are

$$
\begin{aligned}
& \frac{\partial \Gamma_{A}}{\partial t}=-k_{o x} \Gamma_{A}+k_{\text {red }} \Gamma_{B}+k_{\text {ads }}^{A}[A]_{0}\left(\Gamma_{\max }-\Gamma_{A}-\Gamma_{B}\right)-k_{\text {des }}^{A} \Gamma_{A} \\
& \frac{\partial \Gamma_{B}}{\partial t}=k_{o x} \Gamma_{A}-k_{\text {red }} \Gamma_{B}+k_{a d s}^{B}[B]_{0}\left(\Gamma_{\max }-\Gamma_{A}-\Gamma_{B}\right)-k_{d e s}^{B} \Gamma_{B} \\
& \left(\frac{\partial[A]}{\partial t}\right)=D_{A} \frac{\partial^{2}[A]}{\partial x^{2}} \\
& \left(\frac{\partial[B]}{\partial t}\right)=D_{B} \frac{\partial^{2}[B]}{\partial x^{2}} \\
& D_{A}\left(\frac{\partial[A]_{0}}{\partial x}\right)_{x=0}=k_{a d s}^{A}[A]_{0}\left(\Gamma_{\max }-\Gamma_{A}-\Gamma_{B}\right)-k_{d e s}^{A} \Gamma_{A} \\
& D_{B}\left(\frac{\partial[B]_{0}}{\partial x}\right)_{x=0}=k_{a d s}^{B}[B]_{0}\left(\Gamma_{\max }-\Gamma_{A}-\Gamma_{B}\right)-k_{d e s}^{B} \Gamma_{B}
\end{aligned}
$$

where $t$ is the time during the simulation, $x$ is the distance from the electrode surface and $\Gamma_{A / B}$ is the surface coverage of $A$ or $B$.

The electrode kinetics of surface redox processes can be successfully described by the asymmetric Marcus-Hush model to give physical insights such as the 're-organisation energy' which account for the curvature of Tafel slope at high over-potentials. ${ }^{30-33}$ For small applied over-potentials, however, the Tafel slope is typically linear and thus can be described by the semi-empirical Butler-Volmer kinetics $^{34-36}$

$$
\begin{aligned}
& k_{o x}=k_{0} e^{\frac{\beta F}{R T}\left(E-E_{f}^{0}\right)} \\
& k_{\text {red }}=k_{0} e^{\frac{-(1-\beta) F}{R T}\left(E-E_{f}^{0}\right)}
\end{aligned}
$$

where the electron transfer rate constants $k_{o x}$ and $k_{\text {red }}$ varies exponentially with a transfer coefficient $\beta$ and the applied potential. The formal potential $E_{f}^{0}$ is defined as the potential at which 
the surface coverages $\Gamma_{A}=\Gamma_{B}$ at equilibrium (see SI section one). The Butler-Volmer kinetics is used here for ease of computation and to explore the role of adsorption kinetics.

During the cyclic voltammetry simulation, the potential applied to the electrode $E$ varies linearly with time

$$
E=E_{\text {initial }}+v t
$$

here $E_{\text {initial }}$ is the starting potential and $v$ is the scan rate. At time $t=t_{\text {switch }}$, the potential reaches $E_{\text {switch }}$ and the potential sweep changes direction

$$
E=E_{\text {switch }}-v\left(t-t_{\text {switch }}\right)
$$

At the start of the simulation, species $A$ is present uniformly throughout the solution

$$
t=0,0 \leq x \leq \infty\left\{\begin{array}{c}
{[A]_{x}=[A]_{b u l k}} \\
{[B]_{x}=0}
\end{array}\right.
$$

The simulation assumes sufficient time was allowed before any measurement for the surface coverage $\Gamma_{A}$ to reach equilibrium with $[A]_{b u l k}$

$$
t=0\left\{\begin{array}{c}
\Gamma_{A, \text { pre }}-\text { adsorbed } \\
\Gamma_{B, \text { pre-adsorbed }}=\frac{k_{a d s}^{A}[A]_{b u l k} \Gamma_{\max }}{k_{a d s}^{A}[A]_{b u l k}+k_{\text {des }}^{A}}
\end{array}\right.
$$

During the cyclic voltammetry, the current $I$ reflects contribution from the net flux from the rate of the redox processes occurring on the electrode surface

$$
\frac{I}{F A}=j=k_{o x} \Gamma_{A}-k_{\text {red }} \Gamma_{B}
$$

where $F$ is the Faraday constant and $A$ is the area of the electrode. To generalise the simulation results for the broad range of experimental parameters, dimensionless parameters will be used. The required normalisation of the dimensioned parameters is discussed in the next section.

\subsection{Normalised parameters}

Dimensionless parameters will be used to characterise the simulated voltammograms. The latter are presented in the dimensionless form of flux $J$ as a function of dimensionless potential $\Theta$. Table S1 (see supporting information) shows the dimensional parameters which are used for the normalisations shown in Table S2. 
The mathematical model can be expressed in the dimensionless form

$$
\begin{aligned}
& \frac{\partial \theta_{A}}{\partial T}=-K_{o x} \theta_{A}+K_{r e d} \theta_{B}+K_{a d s}^{A} B C_{A, 0}\left(1-\theta_{A}-\theta_{B}\right)-K_{d e s}^{A} B \theta_{A} \\
& \frac{\partial \theta_{B}}{\partial T}=K_{o x} \theta_{A}-K_{r e d} \theta_{B}+K_{a d s}^{B} B C_{B, 0}\left(1-\theta_{A}-\theta_{B}\right)-K_{d e s}^{B} B \theta_{B} \\
& \left(\frac{\partial C_{A}}{\partial T}\right)_{X>0}=\frac{\partial^{2} C_{A}}{\partial X^{2}} \\
& \left(\frac{\partial C_{B}}{\partial T}\right)_{X>0}=d_{B} \frac{\partial^{2} C_{B}}{\partial X^{2}} \\
& \left(\frac{\partial C_{A}}{\partial X}\right)_{X=0}=K_{a d s}^{A} C_{A, 0}\left(1-\theta_{A}-\theta_{B}\right)-K_{d e s}^{A} \theta_{A} \\
& \left(\frac{\partial C_{B}}{\partial X}\right)_{X=0}=\frac{K_{a d s}^{B}}{d_{B}} C_{B, 0}\left(1-\theta_{A}-\theta_{B}\right)-\frac{K_{d e s}^{B}}{d_{B}} \theta_{B}
\end{aligned}
$$

The initial simulation conditions in dimensionless form are shown in Table 1.

\begin{tabular}{lc} 
Table 1 initial simulation conditions. & \\
\hline Equations & Boundary regions \\
\hline$C_{A}=1$ & $0 \leq X \leq \infty, T=0$ \\
$C_{B}=0$ & $0 \leq X \leq \infty, T=0$ \\
$\theta_{A}=\theta_{A, \text { pre-adsorbed }}$ & $T=0$ \\
$\theta_{B}=0$ & $T=0$ \\
\hline
\end{tabular}

where $\theta_{A, \text { pre-adsorbed }}$ is the dimensionless surface coverage of $\mathrm{A}$ at adsorption equilibrium prior to the start of simulation

$$
\theta_{A, \text { pre-adsorbed }}=\frac{K_{a d s}^{A} C_{A, \text { bulk }}}{K_{a d s}^{A} C_{A, b u l k}+K_{\text {des }}^{A}}
$$

The dimensionless potential $\Theta$ varies linearly with dimensionless time $T$ and dimensionless scan rate $\sigma$ similar to equation 3 and 4

$$
\begin{gathered}
\Theta=\Theta_{\text {initial }}+\sigma T \\
\Theta=\Theta_{\text {switch }}-\sigma\left(T-T_{\text {switch }}\right)
\end{gathered}
$$

The dimensionless flux $J$ at the electrode surface is

$$
J=\frac{1}{B}\left(K_{o x} \theta_{A}-K_{r e d} \theta_{B}\right)
$$


where $B$ is a "saturation" parameter that links surface coverage and concentration (see Table S2). The dimensionless flux $J$ can then be converted back to the dimensional current $I$ via

$$
I=\pi r_{e} F D_{A}[A]_{b u l k} J
$$

Next section discusses the approach to solving the set of partial equations subject to the boundary conditions of interest.

\subsection{Numerical simulation and convergence}

The partial differential equations for this one-dimensional system were discretised using the backward implicit method ${ }^{37}$ with a uniform temporal grid and a spatial grid that expands exponentially from the electrode surface to

$$
X_{\max }=6 \sqrt{T_{\max }}
$$

where $T_{\max }$ is the dimensionless duration of the cyclic voltammetry scan and $X_{\max }$ is a dimensionless distance defined well beyond the thickness of the diffusion layer during the cyclic voltammetry. ${ }^{38}$ The sets of discretised diffusion equations were solved using the extended Thomas algorithm. ${ }^{38,39}$

The simulation model was validated with tests that benchmark each constituent part of the model. ${ }^{40}$ For a fully reversible surface-bound redox system, the analytical expression of the dimensional peak

current is $\frac{n^{2} F^{2} v}{4 R T} A \Gamma_{\max }{ }^{41}$ where $n$ is the number of electron transferred and $A$ is the area of the electrode; this can be converted to the dimensionless form to give the expression for peak flux of $\sigma / 4 B$. The surface-bound redox processes in the simulation model was first tested by setting a fast electron transfer rate constant $K_{0}$ relative to the mass transport and all the adsorption and desorption rate constants $\left(K_{a d s}^{A}, K_{d e s}^{A}, K_{a d s}^{B}, K_{d e s}^{B}\right)$ to zero, and the initial surface coverage of $\theta_{A}$ to unity. The simulated peak flux is approximately equal to $\sigma / 4 B$, which agrees with the analytical expression. Second, the diffusion of the species from the bulk were tested by applying an overpotential at $T=0$ with the rate of adsorption and desorption set to a high value such that the mass transport rate to the electrode becomes the rate limiting step; this results in a current transient described by the Cottrell equation. ${ }^{42}$ Finally, the conservation of mass was checked and was conserved throughout all of the simulations. The values of the incremental spatial and temporal point were set to a small value to ensure numerical convergence. The simulations were developed in $\mathrm{C}++$ and ran on an dual Intel (R) Core (TM) E5-2640 v4 CPU $2.40 \mathrm{GHz}$ PC with $16.00 \mathrm{~GB}$ of RAM, with a typical run time of approximately three minutes. 


\section{Results and discussion}

The full model illustrated in Figure 1 contains nine independent variables, namely $K_{0}, K_{\text {des }}^{A}$, $K_{a d s}^{A}, K_{d e s}^{B}, K_{a d s}^{B}, d_{B}, \beta, \sigma$ and $B$. A fully general working surface for the current-voltage curve, reflecting all combinations of those independent variables is clearly neither possible to realistically generate nor useful in the understanding of the curves. Rather for the latter purposes, the model focuses on three limiting cases which reduce the complexity as well as provide meaningful physicochemical insights. In all cases, only species $A$ is present in the bulk solution and equilibrium is reached with the surface coverage of A prior to the voltammetry "experiment" being commenced. Cyclic voltammograms are then simulated with the applied potential varying linearly with time in a triangular wave shape. The differences between the three limiting cases considered are the extent to which the surface bound product $\theta_{B}$ diffuses into the bulk solution; in case one, the $\theta_{B}$ remains on the surface and does not desorb; in case two, $\theta_{B}$ slowly desorbs into solution; in case three, $\theta_{B}$ desorbs rapidly into the solution.

\subsection{Case one - species B remains on the surface}

In the first limiting case, the surface bound species B remains on the surface. A schematic of the simplified processes is shown in Figure 2. The number of independent parameters reduced down to $K_{0}, K_{d e s}^{A}, K_{a d s}^{A}, \beta, \sigma$ and $B$. The work by Chevallier et al. provided a general quantitative view of Case One for a micro-electrode by, effectively, examining all the independent parameters using two to three values for each of the parameters. ${ }^{25}$ In this study, we study in-depth how the adsorption rate constant $K_{a d s}^{A}$, the electron transfer rate constant $K_{0}$ and the scan rate $\sigma$ affect the shape and peak size of the voltammograms. The other parameters are set constant: $K_{\text {des }}^{A}=1, d_{B}=1, \beta=$ 0.5 and $B=100$. 


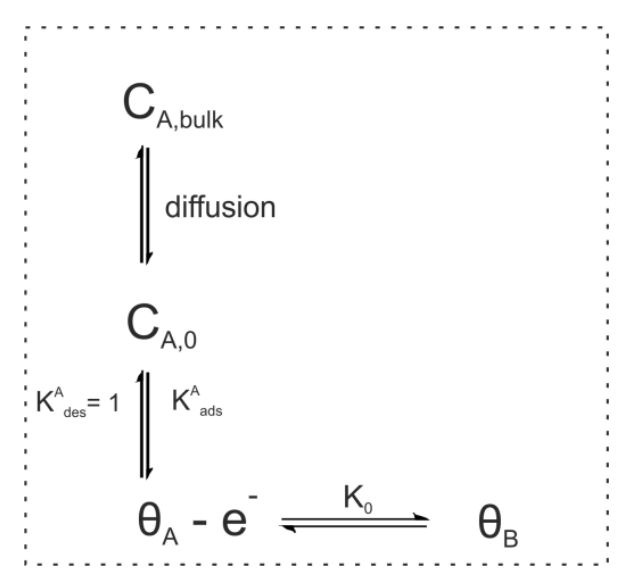

Figure 2. The reaction mechanism for Case One, where the product B is exclusively surface bound.

Figure 3 a) shows the simulated cyclic voltammograms under fast and 'reversible' electron transfer kinetics with different adsorption rate constants $K_{a d s}^{A}$. Note, the term fast or slow electron transfer kinetics in this article is relative to the rate of mass transport from the bulk solution. ${ }^{43}$ The simulation assumes adsorption equilibrium was reached prior to the start of simulation. For a small adsorption rate constant of $\mathrm{A}$, relative to the corresponding desorption constant (i.e. $K_{\text {ads }}^{A} / K_{\text {des }}^{A}<1$ ), only a small fraction of the maximum surface coverage is occupied by A prior to the start of the experiment. During the oxidation sweep, $\theta_{A}$ undergoes oxidation to form $\theta_{B}$ and the equilibrium position between the concentration adjacent to the electrode $C_{A, 0}$ and the coverage $\theta_{A}$ is perturbed. The rapid depletion of the pre-adsorbed $\theta_{A}$ "out runs" the rate of adsorption and mass transport of A which results in a peak on the oxidative sweep. The decay of flux is a result of the total sites being occupied by adsorbed B. At higher $K_{a d s}^{A}$ the peak shape becomes narrower and occurs at lower potential with a quicker decay of flux; this is due to the higher fraction of total surface site $\theta_{A, p r e-a d s o r b e d}$ prior to the experiment.

In the limit of very large adsorption rate constant $\left(K_{\text {ads }}^{A} / K_{\text {des }}^{A} \gg 1\right)$, the fraction of the preadsorbed surface coverage $\theta_{A \text {,pre-adsorbed }}$ approaches unity. The shape of the simulated voltammogram becomes symmetric with a peak flux $J$ equal to $2.5 \times 10^{-3}$ under the simulation condition of $\sigma=1$ and $B=100$. Since the formed product $\theta_{B}$ does not diffuse into solution in this case and all the adsorption sites were initially occupied by $A$, the simulated voltammogram matches exactly to that of a reversible surface-bound redox system. ${ }^{41}$ The latter can be solved analytically to give an expression for the peak current, $\frac{n^{2} F^{2} v}{4 R T} A \Gamma_{\max }$, where $n$ is the number of electron transferred and $A$ is the area of the electrode; in the dimensionless form, the expression of peak flux $J_{\text {peak }}$ for a fully reversible surface bound system is $\sigma / 4 B$, which is in excellent agreement with the simulation result at large $K_{a d s}^{A}$. Figure $3 \mathrm{~b}$ ) shows a plot of the simulated peak flux for different scan rate $\sigma$, 
where $J_{s r}$ is the normalised peak flux $J_{\text {peak }}$ against scan rate $\sigma$. For all simulated $\sigma$, the $J_{s r}$ approaches to $1 / 4 B$ at high $K_{a d s}^{A}$.
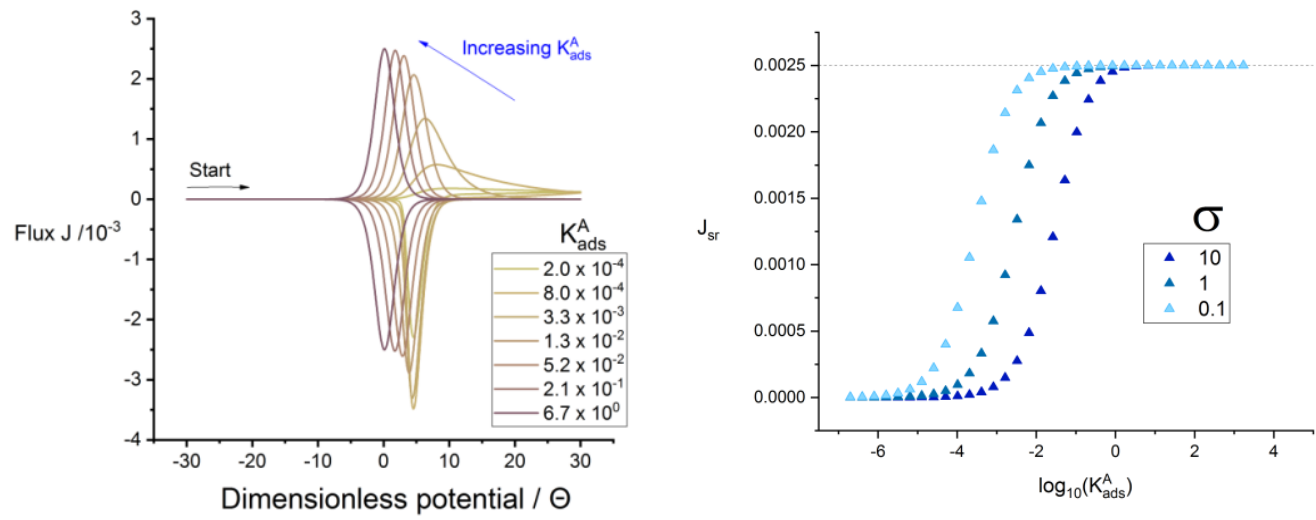

Figure 3. a) Simulated voltammograms of Limiting Case One with different adsorption rate constant of $A$, $K_{a d s}^{A}$, under fast electron transfer kinetics $K_{0}=1 \times 10^{7}$ and $\sigma=1$. b) plot of $J_{s r}$ against the $\log \left(K_{\text {ads }}^{A}\right)$, where $J_{s r}$ is the dimensionless peak flux $J$ normalised against the dimensionless scan rate $\sigma$. Other parameters are $B=100, E_{f}^{0}=0, K_{\text {des }}^{A}=1, K_{\text {ads }}^{B}=0, K_{\text {des }}^{B}=0$ and $d_{B}=1$.

The simulated cyclic voltammogram for Case One with slow electron transfer kinetics, relative to mass transport, is shown in Figure 4 a). In contrast to Case One (fast electron transfer kinetics), the peak-to-peak separation increases due to an over-potential is needed to drive the sluggish electron transfer kinetics. As the adsorption rate constant increases relative to that of desorption, $K_{\text {ads }}^{A} / K_{\text {des }}^{A}$, the peak shape becomes narrower similar to what was observed for fast electron transfer kinetics. Shown in Figure $4 \mathrm{~b}$ ) is the plot of $J_{s r}$ for different $\sigma$ under slow electron transfer kinetics. In the limit of high $K_{a d s}^{A}$, the peak flux $J$ approaches $1.84 \times 10^{-3}$, which is lower compared to the $2.5 \times 10^{-3}$ simulated under fast electron transfer kinetics. This limiting result of $1.84 \times 10^{-3}$ can be derived analytically by considering as $K_{a d s}^{A} \rightarrow \infty$, all the adsorption sites were occupied by A initially with no adsorption during the cyclic voltammetric simulation; therefore, the rate of consumption of $\theta_{A}$ under irreversible electron transfer kinetics is described by

$$
\frac{\partial \theta_{A}}{\partial T}=-K_{o x} \theta_{A}, \quad \text { where } \theta_{A, p r e-a d s o r b e d}=1, K_{o x}=K_{0} e^{\beta \Theta} \text { and } \Theta=\Theta_{\text {initial }}+\sigma T
$$

where $\Theta$ is the applied potential and $\Theta_{\text {initial }}$ is the applied potential at the start of the simulation. Solving this with the boundary condition leads to an expression of the surface coverage $\theta_{A}$ as a function of time $T$ which gives the analytical solution for the peak flux $J, \frac{\beta \sigma}{B} e^{-1}$, which is in excellent agreement with the simulated result; see SI Section Two for more detail. 

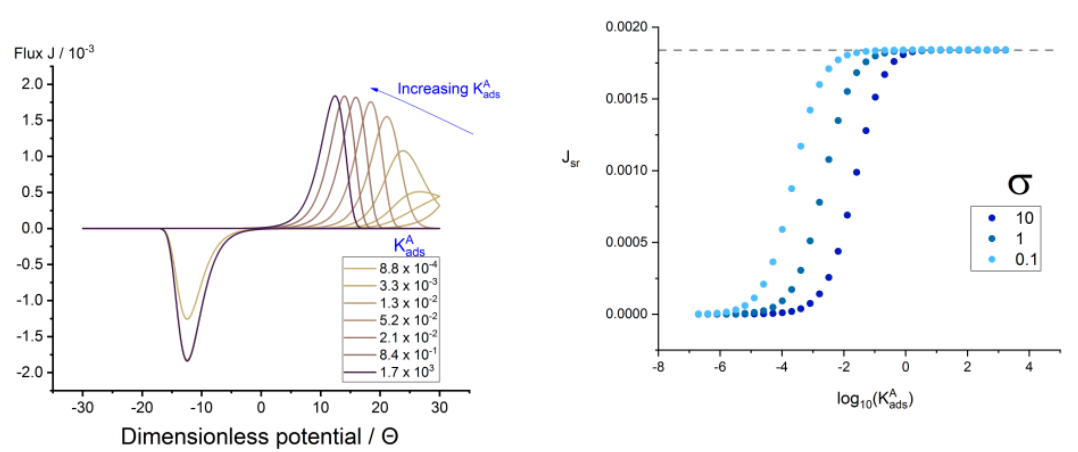

Figure 4. a) Simulated voltammograms of Limiting Case One with different adsorption rate constant of $A, K_{a d s}^{A}$, under slow electron transfer kinetics $K_{0}=1 \times 10^{-3}$ and $\sigma=1$. b) plot of $J_{s r}$ against the $\log \left(K_{\text {ads }}^{A}\right)$, where $J_{s r}$ is the dimensionless peak flux $J$ normalised against the dimensionless scan rate $\sigma$. Other parameters are: $\beta=0.5, B=100, E_{f}^{0}=0, K_{\text {des }}^{A}=1, K_{\text {ads }}^{B}=0, K_{\text {des }}^{B}=0$ and $d_{B}=1$.

Overall and to summarise, Limiting Case One describes how $K_{0}, K_{a d s}^{A}$ and $\sigma$ varies the shape and peak flux of the voltammogram for when species B remains adsorbed. Next, Limiting Cases Two and Three build upon Case One and investigate the simulated voltammograms when species B can diffuse into the bulk solution.

\subsection{Case two - species B is partly released into solution}

In Case Two, the model includes non-zero adsorption and desorption rate constants for $\mathrm{B}$. The schematic of Case Two is shown in Figure 5. Usually but not essentially the rate of desorption of $B$ is set lower than the adsorption of $\mathrm{B}, K_{d e s}^{B}<K_{a d s}^{B}$. This allows the formed redox product $\theta_{B}$ to partially desorb into solution. Herein, the effect of varying $K_{0}, K_{a d s}^{A}$ and $K_{d e s}^{B}$ on the voltammogram will be investigated. The other parameters are set constant: $K_{d e s}^{A}=1, K_{a d s}^{B}=1, d_{B}=1, \sigma=1, \beta=$ 0.5 and $B=100$.

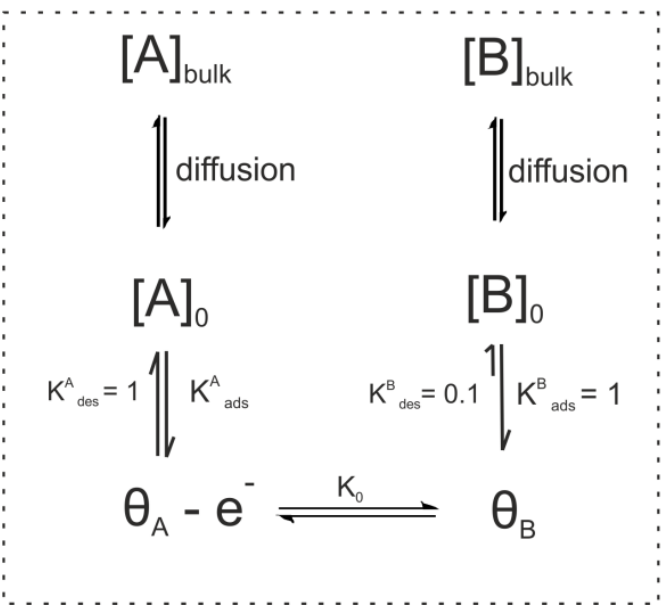

Figure 5. The reaction mechanism for Case 2, where some B slowly diffuses away from the electrode surface.

Figure 6 a) shows the cyclic voltammograms of Case Two under fast surface electron transfer kinetics with different adsorption rate constants for $\mathrm{A}, K_{a d s}^{A}$. At a slow $K_{a d s}^{A}$, an oxidation peak appears on the forward sweep follow by a decay of current. As $K_{a d s}^{A}$ increases, the shape of the 
simulated voltammogram approaches a steady state flux. A peak appears at slow $K_{a d s}^{A}$ due to the fast consumption of the pre-adsorbed $\theta_{A}$ with a gradual decay of flux from the interplay between the rate of adsorption of $A$, mass transport and the decreasing number of unoccupied sites on the electrode. Note the observed flux decay immediately after the oxidative peak is prolonged in Case Two, in contrast to Case One, due to the inclusion of the desorption of B which allows more $A$ to be reduced. Figure 6 b) shows a plot of normalised peak flux $J_{d r c}$ as a function of $K_{a d s}^{A}$ for different magnitude of the desorption rate constant $K_{\text {des }}^{B}$

$$
J_{d r c}=J / K_{d e s}^{B}
$$

where $J_{d r c}$ is the peak flux $J$ normalised against the desorption rate constant $K_{d e s}^{B}$. As the adsorption rate constant of $\mathrm{A}$ is larger than the desorption rate constant of $\mathrm{B}, K_{a d s}^{A} \gg K_{d e s}^{B}$, the flux becomes "bottlenecked" by the slow rate of desorption of B at over-potential. Under these conditions, the rate of desorption of $\mathrm{B}$ is equal to $K_{d e s}^{B} \theta_{B}$ and the flux $J$ approximately equals to the desorption rate constant $K_{\text {des }}^{B}$ (where $\theta_{B} \approx 1$ ). Therefore, $J_{d r c}$ in Figure $6 \mathrm{~b}$ ) approaches unity when $K_{\text {ads }}^{A} \gg K_{\text {des }}^{B}$.

Next, the effect of slow electron transfer kinetics on Case Two is investigated; simulated voltammograms are shown in Figure 7 a). On the forward oxidation sweep, the peak shape resembles that under fast rates of electron transfer. However, the peak position shifts positively as the slower electron transfer kinetics requires a high overpotential to drive the current. Figure $7 \mathrm{~b}$ ) plots the simulated peak flux $J_{d r c}$ against $K_{a d s}^{A}$. As observed under fast electron transfer kinetics, the $J_{d r c}$ approaches unity at high $K_{a d s}^{A}$ as the desorption of B becomes the rate limiting step.
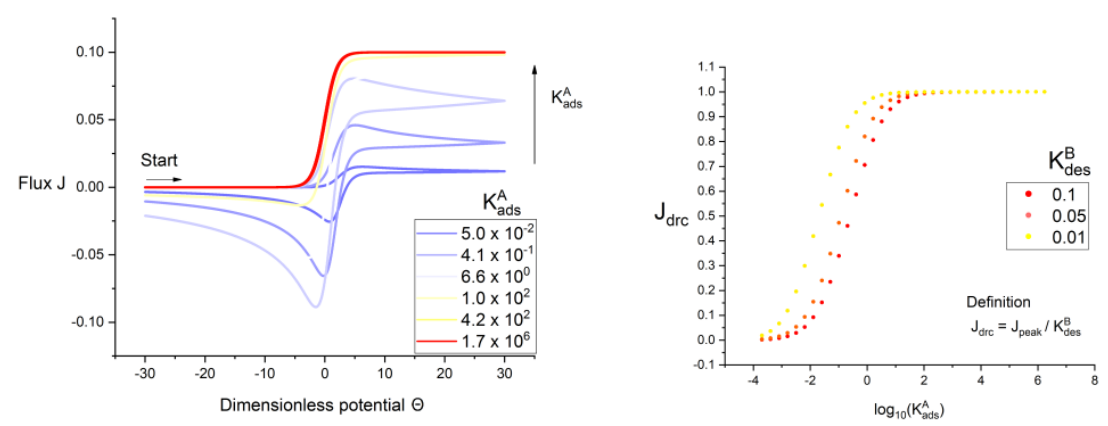

Figure 6. a) Simulated cyclic voltammogram of case two with increasing adsorption rate constant of $A, K_{a d s}^{A}$, under fast electron transfer kinetics $K_{0}=1 \times 10^{7}$ and $K_{\text {des }}^{B}=0.1$. b) Plot of $J_{d r c}$ against $\log \left(K_{\text {ads }}^{A}\right)$ under fast electron transfer kinetics $K_{0}=1 \times 10^{7}$ for different $K_{\text {des. }}^{B}$. Other parameters are: $B=100, \sigma=1, E_{f}^{0}=0, K_{\text {des }}^{A}=1, K_{\text {ads }}^{B}=1$, and $d_{B}=1$. 

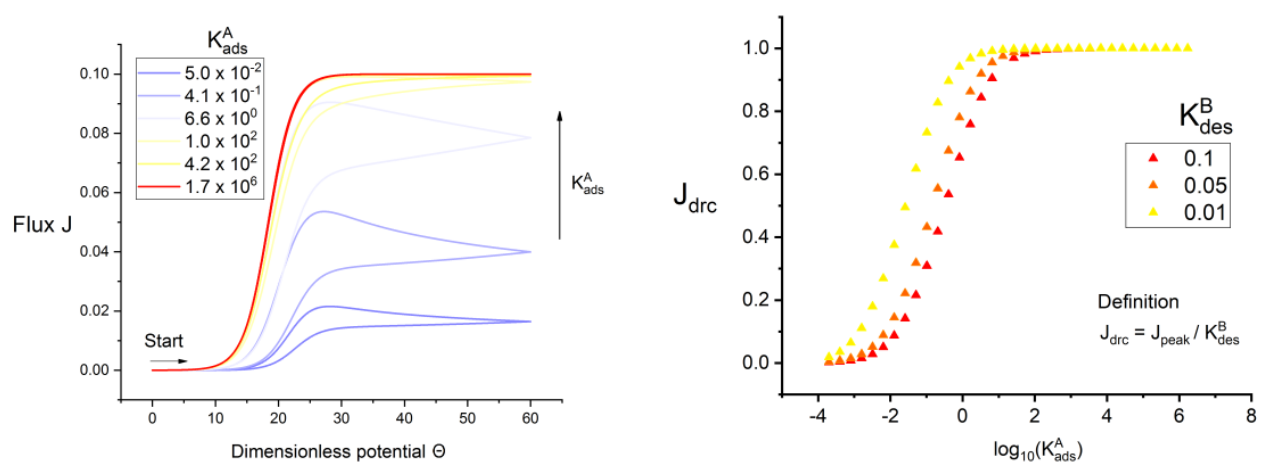

Figure 7. a) Simulated cyclic voltammogram of case two with increasing adsorption rate constant of $A, K_{a d s}^{A}$, under slow electron transfer kinetics $K_{0}=1 \times 10^{-3}$ and $K_{d e s}^{B}=0.1$. b) Plot of $J_{d r c}$ against $\log \left(K_{a d s}^{A}\right)$ under slow kinetics $K_{0}=1 \times 10^{-3}$ for different $K_{d e s}^{B}$. Other parameters are: $B=100, \sigma=1, E_{f}^{0}=0, K_{\text {des }}^{A}=1, \quad K_{\text {ads }}^{B}=1$, and $d_{B}=1$.

In summary, the interesting phenomenon emerge from Case Two when $K_{a d s}^{A}>>K_{d e s}^{B}$. In this case the shape of voltammogram reaches a steady state current - the size of which reflects the rate of desorption of B. The next section will discuss the simulation results for the Limiting Case Three, different to Case Two, the formed redox product $\theta_{B}$ desorbs rapidly into the bulk solution.

\subsection{Case three - species B is substantially released into solution}

In Case Three, the formed redox product $\theta_{B}$ is allowed to desorb quickly into solution. The reaction mechanism is illustrated in Figure 8. The effect of $K_{a d s}^{A}, K_{0}$ and $\sigma$ on the voltammetric shape and peak flux is considered. Other parameters were set constant: $K_{\text {des }}^{A}=1, K_{\text {des }}^{B}=1 \times 10^{6}, K_{a d s}^{B}=1$, $d_{B}=1, \beta=0.5$ and $B=100$.

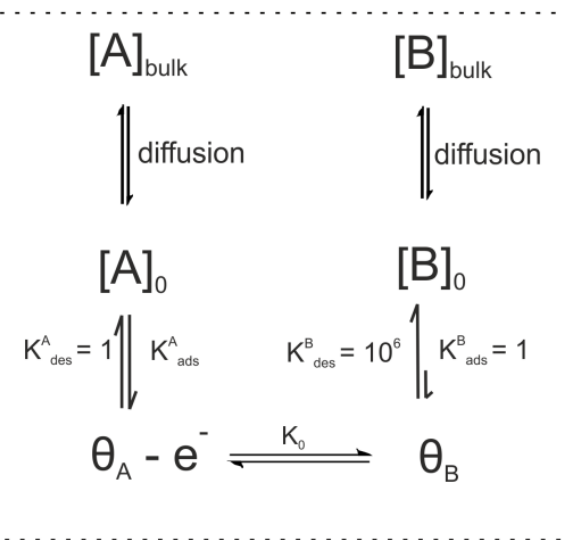

Figure 8. The reaction mechanism for case three, where the adsorbed species B desorbs rapidly after formation.

The voltammograms of Case Three were simulated under fast electron transfer kinetics for different $K_{a d s}^{A}$, as shown in Figure 9 a). The oxidative peak potentials shift negatively due to the rapid desorption of the formed redox product which drives the reaction at low potentials. 
Interesting, as $K_{a d s}^{A}$ increases, the oxidative peak shape becomes narrower. As the rate of adsorption of $\mathrm{A}$ increases to a magnitude comparable to the rapid desorption of $\mathrm{B}\left(K_{\text {ads }}^{A} \approx K_{\text {des }}^{B}=\right.$ $1 \times 10^{6}$ ), the flux $J$ increases sharply on the oxidative sweep before a sudden and rapid decay that leads to a sharp oxidative wave. This is similar to what is observed for the voltammograms simulated under slow electron transfer kinetics, shown in Figure $10 \mathrm{a}$ ). In the limit of a rapid rate of adsorption of $A$ and desorption of $B$, the limiting step in the system becomes the rate of electron transfer and the mass transport of material from the bulk solution. The simulated peak fluxes may be insightfully compared and contrasted to that for a simple solution phase redox system, $A_{(a q)}-e^{-} \rightleftharpoons$ $B_{(a q)}$, where the electron transfer occurs from the solution phase with the peak currents described by the Randles - Ševčík equation. ${ }^{44,45}$ This leads to the normalization of

$$
J_{R S}=J_{\text {peak }} / J_{\text {Randles }- \text { Ševčík }}
$$

where the dimensionless form of the Randles - Ševčík equation in the limit of a fully reversible or fully irreversible electron transfer kinetics can be written as

$$
\begin{aligned}
& J_{r e v, \text { Randles - Ševčík }}=0.446 \sqrt{\sigma} \quad(\text { reversible }) \\
& J_{\text {irrev }, \text { Randles - Ševčík }}=0.496 \sqrt{\beta \sigma} \quad(\text { irreversible })
\end{aligned}
$$

Figure $9 \mathrm{~b}$ ) and Figure $10 \mathrm{~b}$ ) shows a plot of $J_{R S}$ as a function of $K_{a d s}^{A}$ and $\sigma$. Interestingly, for both reversible and irreversible electron transfer kinetics, $J_{R S}$ can exceed unity as $K_{a d s}^{A}$ increases with the former approaches to a value of $1 / 0.446$ and the latter to $1 / 0.496$ - these values are independent of the saturation parameter $B$ for the scan rates investigated. Note, this is not due to the flux contribution from the pre-adsorbed A, since as seen in Case One, the dimensionless peak flux from a pre-adsorbed monolayer of $\mathrm{A}$ is $2.5 \times 10^{-3}$ for the reversible and $1.8 \times 10^{-3}$ for irreversible electron transfer kinetics. This is almost three orders of magnitude smaller than that observed for Case Three, hence the unexpected result of $J_{R S}$ exceeding unity arises solely from mass transport effects from the bulk solution. 

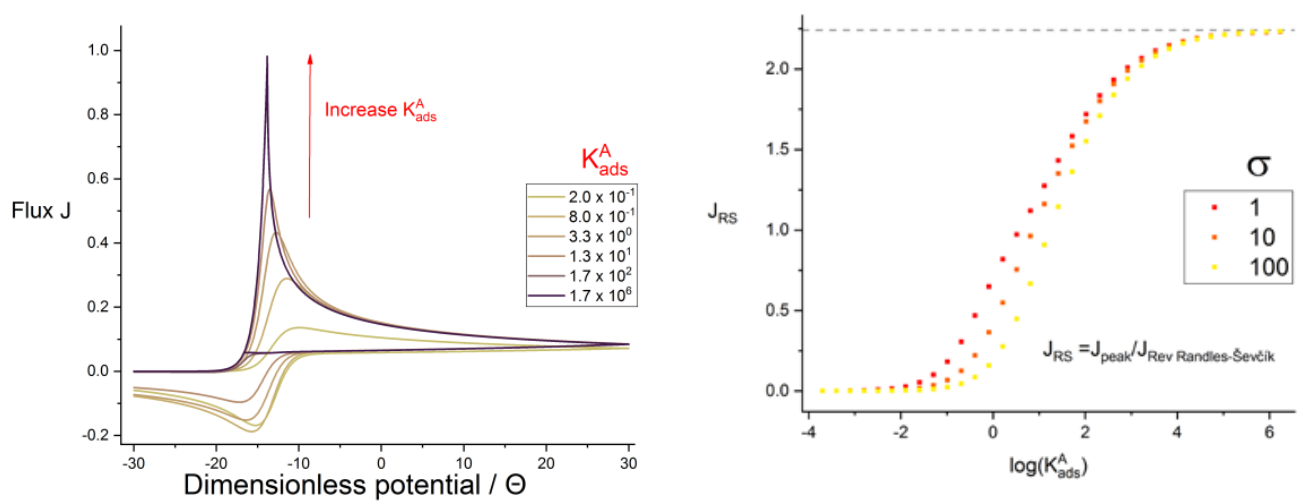

Figure 9. a) Simulated cyclic voltammogram of case three with increasing adsorption rate constant of $A, K_{\text {ads }}^{A}$, under fast electron transfer kinetics $K_{0}=1 \times 10^{7}$ and $\sigma=1$. b) Plot of $J_{R S}$ against $\log \left(K_{\text {ads }}^{A}\right)$ under fast kinetics $K_{0}=1 \times 10^{7}$ for different $\sigma$. Other parameters are: $B=100, \quad E_{f}^{0}=0, \quad K_{\text {des }}^{A}=1, K_{\text {ads }}^{B}=1, K_{\text {des }}^{B}=1 \times 10^{6}$ and $d_{B}=1$.
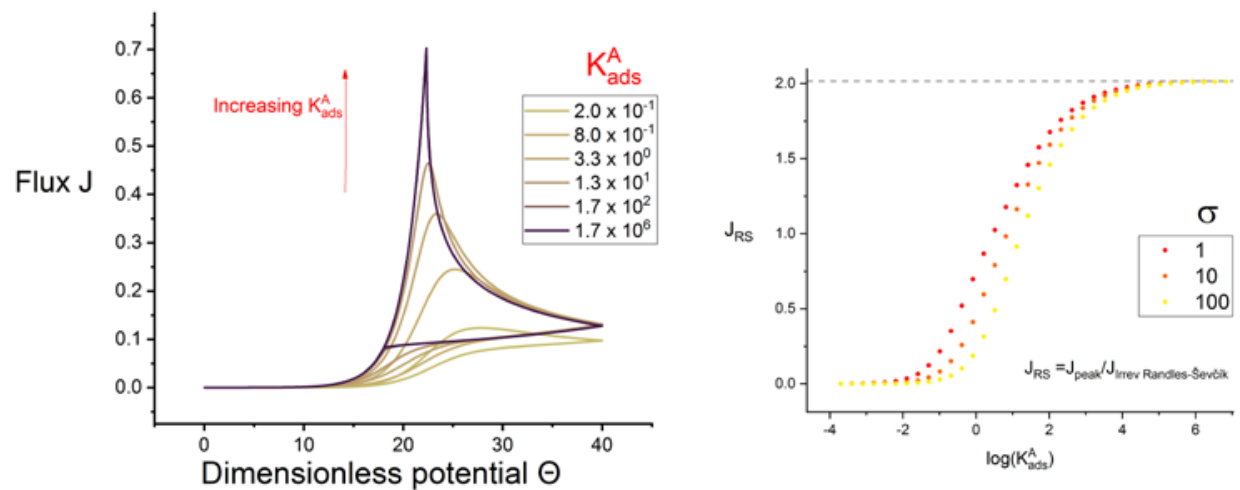

Figure 10. a) Simulated cyclic voltammogram of limiting case three with increasing adsorption rate constant of $A, K_{a d s}^{A}$, under slow electron transfer kinetics $K_{0}=1 \times 10^{-3}$ and $\sigma=1$. B) Plot of $J_{R S}$ against $\log \left(K_{\text {ads }}^{A}\right)$ under slow kinetics $K_{0}=1 \times 10^{-3}$ for different $\sigma$. Other parameters are: $B=100, E_{f}^{0}=0, K_{\text {des }}^{A}=1, K_{\text {ads }}^{B}=1, K_{\text {des }}^{B}=1 \times 10^{6}$ and $d_{B}=1$.

To understand the origin of the sharp peak shape and the discrepancy with the Randles - Ševčík equation, we consider the calculation of the flux $J$ for both cases. For a redox couple that undergoes electron transfer without the need to adsorb, the flux of electrons $J_{\text {solution phase }}$ is a function of the surface concentrations and the rate of electron transfer, described by the Butler-Volmer equation:

$$
J_{\text {solution phase }}=K_{o x} C_{A, 0}-K_{\text {red }} C_{B, 0}
$$

where $C_{A, 0}$ and $C_{B, 0}$ is the concentration of species $\mathrm{A}$ and $\mathrm{B}$ at the electrode surface respectively. For simplification, we will discuss the fully irreversible case where the backward rate $K_{\text {red }}$ is negligible. Under irreversible electron transfer kinetics, $J_{\text {solution phase }}$ is the product of $K_{o x}$ and $C_{A, 0}$. During the oxidative sweep, the rate of oxidation $K_{o x}$ increases exponentially with the applied potential but the surface concentration of $A$ is consumed during this process to form $B$. In contrast, the flux $J$ in the adsorption model under irreversible electron transfer kinetics is the product of $K_{o x}$ and $\theta_{A}$. In the limit of $K_{a d s}^{A} \rightarrow \infty$, the oxidative wave peaks at a dimensionless potential of 22.3; the 
surface coverage of $A$ and $B$, and the concentration profile of $A$ in solution is shown in Figure 11 a) and b) respectively. The time axis in Figure 11 is normalised to the simulation duration, where the oxidation peak potential $\Theta_{\text {peak }}$ occurs at a dimensionless time $T$ of 0.27 . The plot of surface coverage shows that $\theta_{A}$ is maintained at unity from the start of the simulation up until $T=0.27$, which is the moment when the surface concentration $C_{A, 0}$ depletes to zero. Thus the exponential increase in flux $J$ during the oxidation sweep at high $K_{a d s}^{A}$ is the result of $\theta_{A}$ maintained at unity until the depletion of $C_{A, 0}$. Figure 12 shows the simulated $\mathrm{CV}$ of Case Three with $K_{a d s}^{A}$ equal to $1.7 \times 10^{6}$ under irreversible electron transfer kinetics. The exponential increase in flux is in excellent agreement with the calculated $K_{O X}$ as a function of the dimensionless potentials $\Theta$, overlaid as circles. Thus, in the limit of high $K_{a d s}^{A}$ and $K_{\text {des }}^{B}$, a redox couple that undergoes electron transfer via adsorption can result a peak current ca. 2.3 times larger than that undergoes electron transfer in the solution phase; a surprising but impactful result from the simulation.
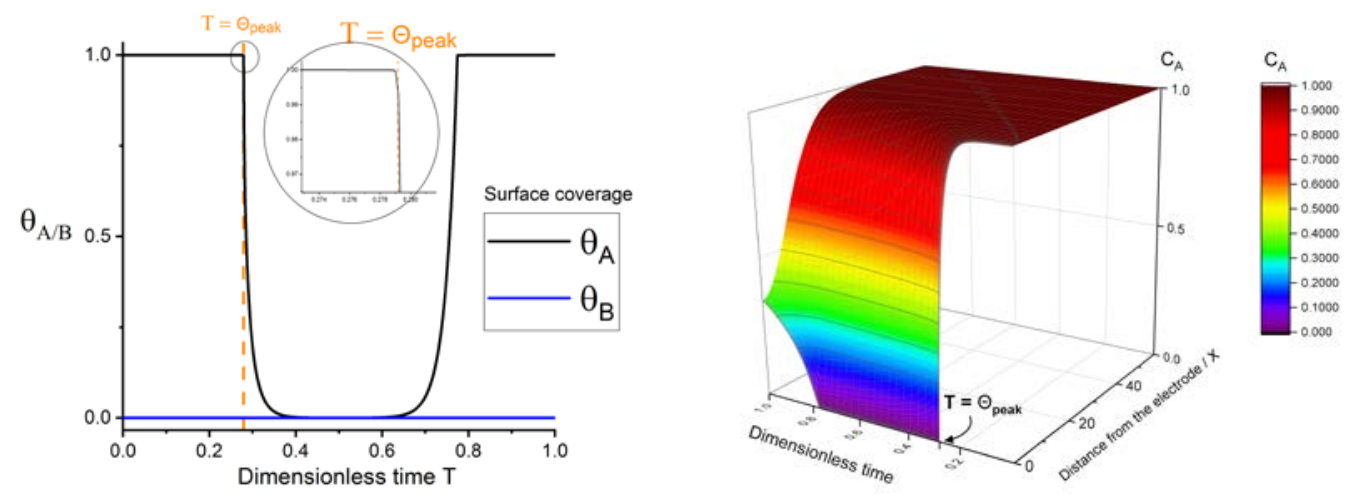

Figure 11. a) The surface coverage profile of $A$ and $B$, and $b$ ) concentration profile of $A$. Both plots were obtained during the simulation of case three under irreversible kinetics and $K_{a d s}^{A}=1.7 \times 10^{6}$. Other parameters are: $B=100, E_{f}^{0}=0, K_{d e s}^{A}=1.7 \times 10^{6}, K_{a d s}^{B}=1, K_{d e s}^{B}=$ 1 and $d_{B}=1$.

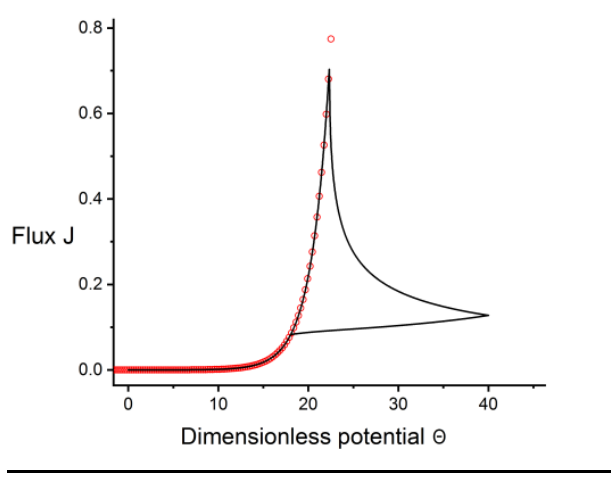

Figure 12. Black line - the simulated cyclic voltammogram for Case Three under irreversible kinetics and $K_{\text {ads }}^{A}=1.7 \times 10^{6}$. Red circles calculated values of $K_{\text {ox }}$ as a function of potential. 


\subsection{Diagnosis of different limiting adsorption cases: a summary}

The voltammetric shape and peak current in the three cases discussed above, in which the extent of product desorption differs, are distinctively different for each of the scenarios. Simulated voltammograms can be used to identify different limiting adsorption cases from experiment. In Case One, the product remains on the surface which results a symmetric peak shape, characteristic of surface bound voltammetry. In Case Two, when desorption of the product becomes the rate determining step, the current approaches an apparent steady state. In Case Three, the voltammetric shape is abnormally sharp when the adsorption kinetics are fast and not rate determining; the resulting flux can be up to 2.3 times bigger in contrast to electron transfer that occurs in the solution phase. In addition we note that the saturation parameter $B$ is a function of the bulk concentration of the reactant allowing easy control over the parameters with the possibility that a change of concentration in the experiment may lead to a switch to a different adsorption mechanism.

\section{Conclusions}

The adsorption model provides new insights to the voltammetric behaviours of surface bound redox systems. The developed model embraces all of the adsorption kinetics, mass transport and electron transfer kinetics of the reactant and products; under certain conditions, this leads to a number of interesting hitherto unexpected current-voltage responses. Most noticeably, the one-dimensional simulation for a macro-electrode reaches a steady state current when rate determining step becomes 'bottlenecked' by the slow desorption of the product. Furthermore, when the rate of adsorption and desorption of the reaction and product are fast and not rate determining, the simulated current for the redox process via the adsorption pathway can be ca. 2.3 times higher to that when electron transfer occurs via the solution phase.

\section{Acknowledgement}

The research leading to these results has received partial funding via an EPSRC Industrial CASE award (EP/N509711/1) in conjunction with Schlumberger (Houston, Texas, United States).

\section{References}

(1) Damaskin, B. B.; Petrii, O. A.; Batrakov, V. V. 1971.

(2) Parsons, R. Surface Science 1980, 101, 316-326. 
(3) Yang, M.; Batchelor - McAuley, C.; Moreira Goncalves, L.; Lima, C. F.; Vyskočil, V.; Tschulik, K.; Compton, R. G. Electroanalysis 2016, 28, 197-202.

(4) Neumann, C. C.; Batchelor - McAuley, C.; Downing, C.; Compton, R. G. Chemistry - A European Journal 2011, 17, 7320-7326.

(5) Yardım, Y.; Gülcan, M.; Şentürk, Z. Food chemistry 2013, 141, 1821-1827.

(6) Ngamchuea, K.; Hurst, P.; Batchelor-McAuley, C.; Compton, R. G. Sensors and Actuators B: Chemical 2016, 232, 138-142.

(7) Chaisiwamongkhol, K.; Ngamchuea, K.; Batchelor-McAuley, C.; Compton, R. G. Analyst 2016, 141, 6321-6328.

(8) Kachoosangi, R. T.; Wildgoose, G. G.; Compton, R. G. Analyst 2008, 133, 888-895.

(9) Laviron, E. Journal of Electroanalytical Chemistry 1995, 382, 111-127.

(10) Frumkin, A.; Butterworths, London, 1964.

(11) Delahay, P. Double layer and electrode kinetics; John Wiley \& Sons Inc, 1965.

(12) Laviron, E. Bull. Soc. Chim. France 1967, 3717.

(13) Laviron, E. Electrochim. Acta 1971, 16, 409.

(14) Laviron, E. J. Electroanal. Chem. 1972, 34, 463.

(15) Laviron, E. Journal of Electroanalytical Chemistry and Interfacial Electrochemistry 1972, 35, 333342.

(16) Laviron, E. Journal of Electroanalytical Chemistry and Interfacial Electrochemistry 1980, 109, 5767.

(17) Laviron, E.; Meunier-Prest, R. Journal of Electroanalytical Chemistry 1994, 375, 79-87.

(18) Laviron, E. Journal of Electroanalytical Chemistry and Interfacial Electrochemistry 1981, 124, 1933.

(19) Laviron, E. Journal of Electroanalytical Chemistry and Interfacial Electrochemistry 1982, 140, 247-268.

(20) Laviron, E. Journal of Electroanalytical Chemistry and Interfacial Electrochemistry 1979, 97, 135149.

(21) Laviron, E. Journal of Electroanalytical Chemistry and Interfacial Electrochemistry 1979, 105, 2534.

(22) Laviron, E. Journal of Electroanalytical Chemistry and Interfacial Electrochemistry 1979, 105, 3542.

(23) Lelievre, D.; Plichon, V.; Laviron, E. Journal of Electroanalytical Chemistry and Interfacial Electrochemistry 1980, 112, 137-145.

(24) Atek, I.; Maye, S.; Girault, H. H.; Affoune, A. M.; Peljo, P. Journal of Electroanalytical Chemistry 2018, 818, 35-43.

(25) Chevallier, F. G.; Klymenko, O. V.; Jiang, L.; Jones, T. G.; Compton, R. G. Journal of Electroanalytical Chemistry 2005, 574, 217-237.

(26) Ji, X.; Chevallier, F. G.; Clegg, A. D.; Buzzeo, M. C.; Compton, R. G. Journal of Electroanalytical Chemistry 2005, 581, 249-257.

(27) Langmuir, I. Am. Chem. Soc. 1918, 40, 1361-1403.

(28) Frumkin, A. Zeitschrift für Physik 1926, 35, 792-802.

(29) Nikitas, P. Journal of the Chemical Society, Faraday Transactions 1: Physical Chemistry in Condensed Phases 1985, 81, 1767-1787.

(30) Marcus, R. A.; Sutin, N. Biochimica et Biophysica Acta (BBA)-Reviews on Bioenergetics 1985, 811, 265-322.

(31) Hush, N. Journal of Electroanalytical Chemistry 1999, 460, 5-29.

(32) Henstridge, M. C.; Laborda, E.; Compton, R. G. Journal of Electroanalytical Chemistry 2012, 674, 90-96.

(33) Laborda, E.; Henstridge, M. C.; Batchelor-McAuley, C.; Compton, R. G. Chemical Society Reviews 2013, 42, 4894-4905.

(34) Butler, J. Transactions of the Faraday Society 1924, 19, 729-733. 
(35) Butler, J. Transactions of the Faraday Society 1924, 19, 734-739.

(36) Erdey-Grúz, T.; Volmer, M. Zeitschrift für physikalische Chemie 1930, 150, 203-213.

(37) Euler, L. Institutionum calculi integralis, 1768; Vol. 1.

(38) Compton, R. G.; Laborda, E.; Ward, K. R. Understanding voltammetry: simulation of electrode processes; World Scientific, 2014.

(39) Thomas, L. H. Watson Sci. Comput. Lab. Rept. 1949.

(40) Kätelhön, E.; Compton, R. G. Analyst 2015, 140, 2592-2598.

(41) Compton, R. G.; Batchelor-McAuley, C.; Dickinson, E. J. F. Understanding Voltammetry: Problems and Solutions; Imperial College Press, 2012.

(42) Cottrell, F. Z Physik Chem 1902, 42, 385.

(43) Matsuda, H.; Ayabe, Y. Zeitschrift für Elektrochemie, Berichte der Bunsengesellschaft für physikalische Chemie 1955, 59, 494-503.

(44) Randles, J. E. Transactions of the Faraday Society 1948, 44, 327-338.

(45) Ševčik, A. Collection of Czechoslovak Chemical Communications 1948, 13, 349-377. 